\title{
Acoustics of Cubic Bubbles: Six Coupled Oscillators
}

\author{
Maxime Harazi, Matthieu Rupin, Olivier Stephan, Emmanuel Bossy, and Philippe Marmottant๑* \\ Univ. Grenoble Alpes, CNRS, LIPhy, F-38000 Grenoble, France
}

(Received 12 April 2019; revised manuscript received 30 August 2019; published 16 December 2019)

\begin{abstract}
We introduce cubic bubbles that are pinned to 3D printed millimetric frames immersed in water. Cubic bubbles are more stable over time and space than standard spherical bubbles, while still allowing large oscillations of their faces. We find that each face can be described as a harmonic oscillator coupled to the other ones. These resonators are coupled by the gas inside the cube but also by acoustic interactions in the liquid. We provide an analytical model and 3D numerical simulations predicting the resonance with very good agreement. Acoustically, cubic bubbles prove to be good monopole subwavelength emitters, with nonemissive secondary surface modes.
\end{abstract}

DOI: 10.1103/PhysRevLett.123.254501

Introduction.-Spherical air bubbles in water are known to be excellent acoustic resonators $[1,2]$ because of the important compressibility of the gas compared to that of water, allowing a large amplitude of vibration. Furthermore, the wavelength at resonance is far bigger (500 times) than the bubble size. Bubbles are therefore good candidates for the design of new acoustic metamaterials including bubbles as subwavelength building blocks. Such metamaterials show an enhanced absorption at resonance $[3,4]$ or feature remarkable properties such as a negative index of refraction [5-7] or the ability to focus acoustic energy with a subwavelength precision [8].

If large vibration amplitudes are possible in water, the experimentalist is quickly facing two important issues with spherical bubbles in water. First their stability in space is not insured because of buoyancy [9], meaning that bubbles need to be kept under a surface or a net before acoustic excitation [10]. Another solution is to include bubbles in a gel [3], but care has to be taken to choose a very soft gel and to not add elastic effects. A second major challenge is the dissolution of gas in the liquid. Indeed the curvature of the surface is responsible for a capillary overpressure that triggers dissolution, even into equilibrated or oversaturated water [11]. Stability analysis shows that a spherical bubble is thus always unstable in the long term.

Here we would like to introduce cubic bubbles, designed in order to overcome the issues of stability in position and in size while still performing a large amplitude of vibrations. Our approach is to pin the bubbles within 3D printed frames, resulting in bubbles with flat faces, and therefore no capillary overpressure.

Methods.-The 3D printed frames are crafted in a photoresist polymer with a stereolithographic technology (Kudo3D, $50 \mu \mathrm{m}$ in horizontal resolution and $100 \mu \mathrm{m}$ in vertical resolution). We chose the simplest shape for these frames: a cube [Fig. 1(a)]. The interior size of the cube is $2 a \in[0.9-2.1 \mathrm{~mm}]$, and the edge size $e=0.5 \mathrm{~mm}$.

The frames are then silanized for 20 min using vaporphase deposition of trichloro(perfluorooctyle)silane. This renders the photoresist hydrophobic and enhances the stability of the bubbles over time.

When slowly immersed in deionized water (equilibrated with the ambient atmosphere), a volume of air is trapped in the frame, and six water-air interfaces are created [Fig. 1(b)]. The solid surface being hydrophobic, the interface usually attaches on the external corners, with a roughly flat surface [the blue continuous lines in Fig. 1(c)]. Sometimes the interfaces attached on the inner corners;

(a)

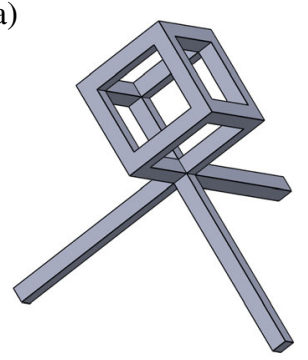

(b)

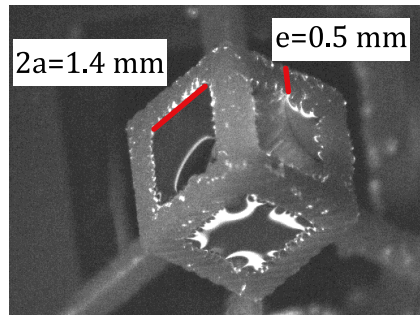

(c)

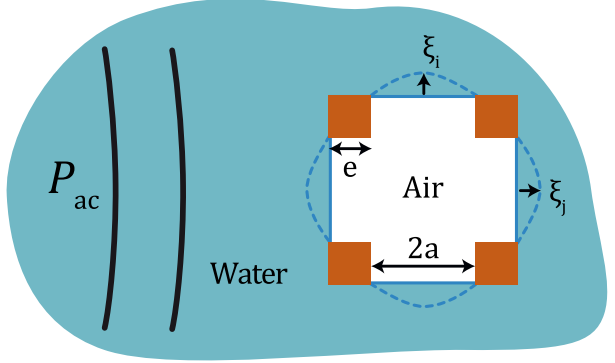

FIG. 1. (a) Design of a cubic frame (the holding pedestal plate not represented). (b) Top view of the immersion of the cubic frame in water: a gas bubble is trapped, and interfaces are visible on each face. (c) Schematic drawing of a cross section of the cube, showing the amplitude of vibration $\xi_{i}$ of an interface labeled $i$, while other faces $j$ have an amplitude $\xi_{j}$. 
these bubbles were discarded. Acoustic measurements are performed right after immersion. Bubbles are then excited in a tank $(30 \times 20 \times 20 \mathrm{~cm})$ using an underwater loudspeaker (Visaton, model FR13WP) driven by one period of sinusoid centered at $2.6 \mathrm{kHz}$, with a typical pressure of $100 \mathrm{~Pa}$, low enough to drive only a linear response. A hydrophone (Brüel \& Kjæ $\mathrm{r}$ 8103) records the pressure signal $P(t)$ in the presence of the bubble, and $P^{0}(t)$ in the absence of the bubble, which allows us by substraction to extract the signal emitted by the bubble only [Fig. 2(a)]. The resonance frequencies of the bubbles were then extracted by computing the Fourier transform of the pressure signals and writing the relative bubble contribution,

$$
\hat{A}(f)=\frac{\hat{P}(f)-\hat{P}^{0}(f)}{\hat{P}^{0}(f)},
$$

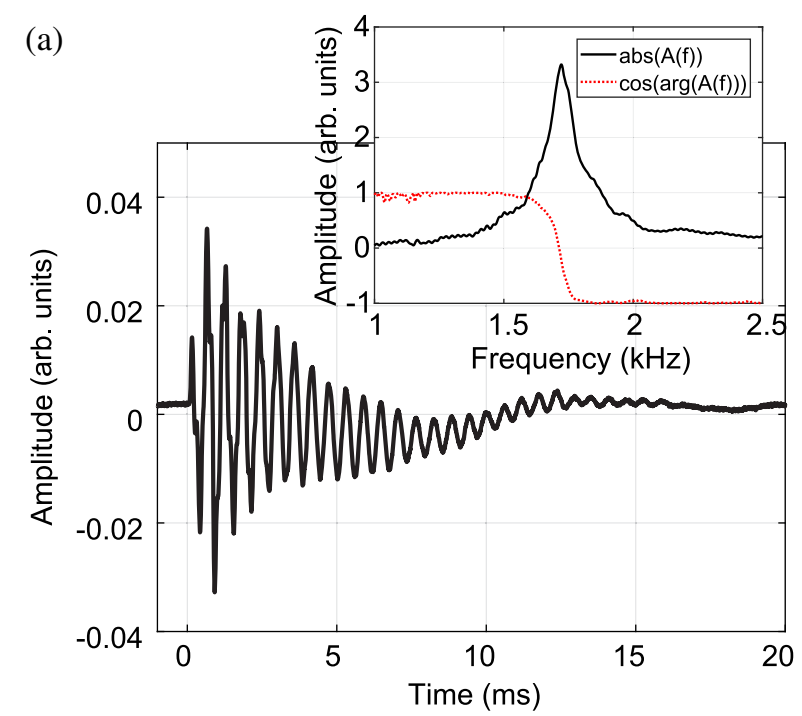

(b)

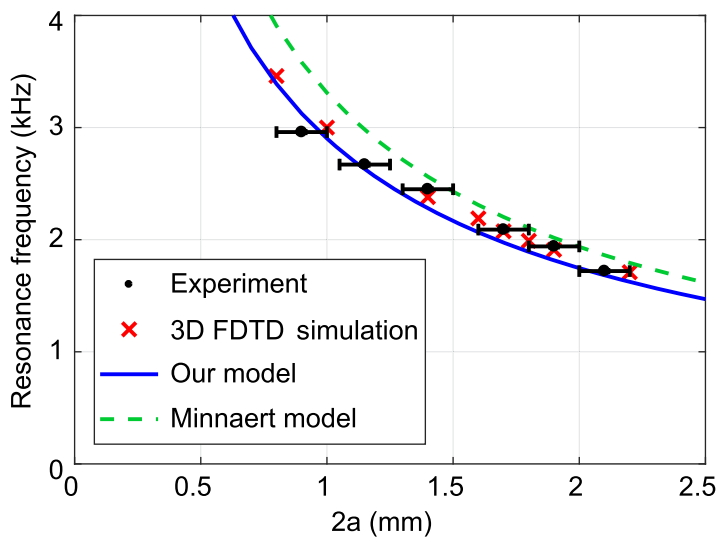

FIG. 2. (a) Signal emitted by a bubble $(2 a=2.1 \mathrm{~mm})$ after a pulse excitation. (Inset) Frequency spectrum of the signal emitted, normalized by the signal without a bubble, showing the norm and the cosine of the phase. (b) Measured frequency when varying the inner space between pillars $2 a$, keeping $e=0.5 \mathrm{~mm}$ fixed. Crosses, 3D simulation; line, theory for a cubic bubble with six coupled oscillators [Eq. (5)]; dashes, Minnaert frequency for a bubble of the same gas volume. and looking for the frequency $f=f_{\text {res }}$ such that $|\hat{A}(f)|$ is maximal [inset of Fig. 2(a)].

Stability.-Having designed cubes of differing lengths, we found that air bubbles are trapped if the opening length does not exceed $2 a_{\max }=2.1 \mathrm{~mm}$. Beyond this length, water enters the frame. This is explained simply by the fact that at larger lengths the hydrostatic pressure induced by gravity overcomes surface tension $\sigma$, preventing water from entering. The crossover occurs for a size of the order of the capillary length $l_{c}=\left(\sigma / \rho_{l} g\right)^{1 / 2} \simeq 2.7 \mathrm{~mm}$, which is indeed in the millimetric range of our observations. Because the interfaces are pinned to the exterior of the edges after immersion [Fig. 1(b)], they are roughly flat, canceling any capillary overpressure, contrary to spherical bubbles. We observe that cubic bubbles can easily live more than a few days in still water without any temperature control or dissolved gas concentration control. After a few hours, we observe a slight tendency for interfaces to enter into the frames, thus reducing the gas volume.

Acoustic oscillations.-We wanted to test whether those bubbles were as good resonators as spherical bubbles, in spite of the rigid frame on which interfaces are pinned. Such a frame potentially restricts the oscillation amplitude. Under pulse excitation, we observed that cubic bubbles behave as clear acoustic resonators in the audible range [Fig. 2(a)], with a well-defined resonance frequency and a quality factor $Q \simeq 20$, slightly lower than that of spherical bubbles of similar size $(Q \simeq 35)$ [12]. The vibration amplitudes at the excitation pressure were minute and smaller than $10 \mu \mathrm{m}$, confirming a linear acoustic regime. Experiments varying the aperture length $2 a$ with a fixed pillar size $e$ show that this resonance frequency increases with smaller size [Fig. 2(b)].

A crude model can be made assuming that the volume of gas [inner space calculated using the cube dimensions $\left.V_{g}=(2 a)^{3}+6(2 a)^{2} e\right]$ can be reshaped into an equivalent sphere of radius $R_{\text {eq }}$ given by $V_{g}=4 \pi R_{\text {eq }}^{3} / 3$. The Minnaert formula for resonance of a spherical bubble [1], $f=\alpha / R_{\mathrm{eq}}$ with $\alpha \simeq 3.24 \mathrm{~m} / \mathrm{s}$, is then a first approximation, although it overestimates the experimental results.

Here our goal is to present an analytical model that takes fully into account the geometry dictated by the cube edges. Such a model should predict the effect of varying the number of open faces. We will present also a numerical calculation of the propagation of sound through this complex geometry to consolidate our analytical predictions. We will see that the presence of the cubic frame modifies the frequency, and it is therefore not just a matter of the volume of the gas.

Model for a cubic bubble: Six coupled oscillators.Each of the six interfaces can be modeled as an individual oscillator, with a mass $m$ and a stiffness $k$. For this purpose, we describe the displacement of interfaces monitored by the displacement at the center of the interface $\xi_{i}$ [see Fig. 1(c)], which is assumed to be small in front of the 
bubble size, $\xi \ll a$. With an interface shape assumed to be parabolic (and thus with a homogeneous curvature inducing a homogeneous Laplace pressure jump), the average displacement over the surface is therefore $\bar{\xi}_{i}=\xi_{i} / 2$.

According to Refs. $[13,14]$, the reaction force necessary to set the liquid into motion just in front of the opening is $m \ddot{\bar{\xi}}_{i}$, giving the added mass per interface $m=\frac{32}{15} \rho_{l} a^{3}$. Here we assume that the interface is circular and of radius $a$, thus neglecting the corners, and that the aperture is embedded in an infinite plane. In addition, the interface displacement gives rise to two elastic restoring forces. The first one is $-k_{g} \bar{\xi}_{i}$, a restoring stiffness due to the gas compression $k_{g}=\kappa P_{0}\left(\pi a^{2}\right)^{2} / V_{g}$, with $\kappa$ being the polytropic exponent of the gas (close to the adiabatic specific heat ratio for millimetric bubbles), $P_{0}$ the gas pressure, and $V_{g}$ the volume of gas trapped. Here we assumed the other interface to remain immobile. The second force is $-k_{\sigma} \bar{\xi}_{i}$, with $k_{\sigma}=$ $8 \pi \sigma$ being a capillary term due to the change in surface area. Tackling this with millimetric dimensions, we find that $k_{\sigma} / k_{g} \sim 10^{-2}$ and that capillary forces can be safely neglected, and the stiffness is simply $k=k_{g}+k_{\sigma} \simeq k_{g}$.

A single interface is therefore a mass-spring system with a resonance frequency $\left(k_{g} / m\right)^{1 / 2} / 2 \pi$. To verify this prediction, we designed a variation of our cubic bubble adding solid walls instead of the openings, resulting in $N$ open faces with mobile interfaces and $6-N$ walls. We find that there is very good agreement between this prediction and the experiment with only one open face $[N=1$, the first point of the curve in Fig. 3(a)].

By opening an increasing number $N$ faces (chosen to be adjacent), we observe that the frequency depends strongly on $N$ [Fig. 3(a)]. This suggests that faces are not independent, but instead coupled, oscillators.

We found that these oscillators excited by the incoming acoustic pressure $P_{\text {ac }}$ are actually coupled through the gas and the liquid. The couplings are (1) a gas coupling because when other faces $j$ also move, they compress the common volume of gas, with each face changing the gas pressure by $-k_{g} \bar{\xi}_{j} / \pi a^{2}$, and (2) an acoustic coupling through liquid because other faces generate an oscillating flux $Q=\pi a^{2} \dot{\xi}_{j}$, resulting in a monopolar acoustic pressure that decays with the distance to the center of the face $r$ : $P=\rho_{l} \dot{Q} / 4 \pi r=\rho_{l} a^{2} \ddot{\xi}_{j} / 4 r$ (the near-field limit) and modifying the pressure on other openings. With the size being small compared to the wavelength, we neglect retardation effects and retain the far-field monopolar expression.

Overall, if we write the sum of forces acting on each interface labeled $i$ (displacing the effective mass $m$ ), we obtain the following set of coupled harmonic oscillators,

$$
m \ddot{\xi}_{i}+k_{g} \bar{\xi}_{i}=-\pi a^{2} P_{\mathrm{ac}}-\sum_{j \neq i}\left(k_{g} \bar{\xi}_{j}+\pi a^{2} \rho_{l} a^{2} \frac{1}{4 r_{i j}} \ddot{\xi}_{j}\right)
$$
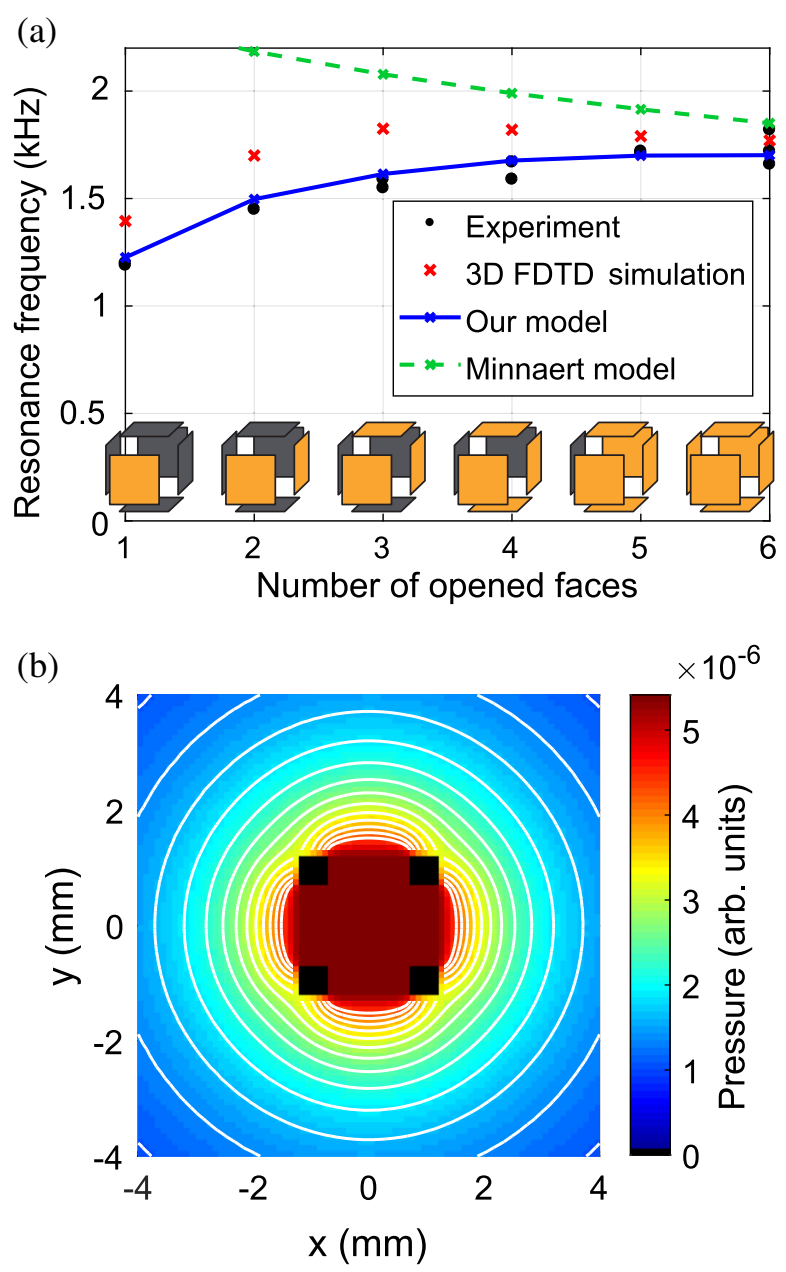

FIG. 3. (a) Resonance frequency as a function of the number of open faces (orange stands for open faces). Lines express the full theory (continuous line) and Minnaert approximation (dashes), $2 a=2.1 \mathrm{~mm}$. Crosses, 3D simulation. (b) Pressure scattered by a cubic bubble $(2 a=1.4 \mathrm{~mm})$ : here an instantaneous cross-section snapshot computed with a 3D finite-difference time-domain (FDTD) simulation in which the bubble oscillates at its natural resonance frequency after a wideband pulse excitation.

with $P_{\text {ac }}$ being the applied acoustic pressure and $r_{i j}$ the distance between the centers of faces $i$ and $j$.

Because of the rotational symmetry of faces on a cube, all faces are equivalent, leading to a distribution of the distances $r_{i j}$ between one face $i$ and the five other ones independent of $i$. The last term on the right-hand side of Eq. (2) is therefore independent of the face number $i$ for $N=6$ (with a restriction $N<6$ that we will discuss just below). Under the same applied pressure, it is reasonable to consider that all faces oscillate with the same amplitude and phase: $\bar{\xi}_{i}=\bar{\xi}_{j}$. The previous equation becomes that of a simple harmonic oscillator with an effective mass

$$
m_{\mathrm{eff}}=m+\pi \rho_{l} a^{3} \sum_{j \neq i} \frac{a}{4 r_{i j}}
$$


and an effective stiffness

$$
k_{\text {eff }}=N k_{g}
$$

since all oscillators compress the same gas volume, demultiplying the resulting overpressure.

On a cube geometry, the distances between the centers of adjacent faces is $r_{i j}=\sqrt{2}(a+e)$. Since waves travel in the liquid, we found it more appropriate to take the shortest path in the liquid, that is $r_{i j}=2(a+e)$. If only $N$ faces are open (chosen to be adjacent), it is straightforward to find by geometry that a good approximation of Eq. (3) is $m_{\text {eff }}=$ $m+(N-1) \Delta m \quad$ with $\quad \Delta m=\pi \rho_{l} a^{3}[a / 8(a+e)]$. This approximation is exact when $N=1,2,3$ adjacent faces, and a slight deviation occurs when $N=4,5,6$ because of the presence of an opposite face distant from $r_{i j}=$ $4(a+e)$ in terms of the liquid path. Note that strictly speaking this opposite face breaks the rotational symmetry for $N=4,5$ since not all faces have the same coupling terms (depending on $r_{i j}$ ) as the other ones, and the single oscillator model is not valid anymore.

The resonance frequency of the coupled oscillators is $\left(k_{\text {eff }} / m_{\text {eff }}\right)^{1 / 2} / 2 \pi$ and is written

$$
f_{\text {res }}=\frac{1}{2 \pi} \sqrt{\frac{N \kappa P_{0} \pi^{2} a^{4} / V_{g}}{\frac{32}{15} \rho_{l} a^{3}+(N-1) \Delta m}} .
$$

Note that the gas volume is given by the inner volume plus the space between the pillars, $V_{g}=(2 a)^{3}+N(2 a)^{2} e$.

Equation (5) is the main prediction of this Letter: it gives the resonance frequency [Fig. 2(b)], with better agreement for small $2 a / e$ aperture ratios than in the Minnaert prediction. For larger $2 a / e$ ratios, the Minnaert prediction is a better approximation, which is consistent with the fact that our model includes the mass $m$ for a vibration of an interface embedded in an infinite plane.

With this formula, we can predict in addition the effect of a varying number of open faces plotted on the experimental curve [Fig. 3(a), line]; we find that it works with no fitting parameter. The agreement is excellent for a small number of openings, while the Minnaert model is clearly out of scope for small $N$ numbers. Note that the gas volume increases slightly with $N$ since the solid walls are open, explaining the decrease of the Minnaert prediction [Fig. 3(a), dashes].

The present arrangement with $N=6$ provides a remarkable rotational symmetry that ensures that all interface oscillators are equivalent (namely, the system is symmetric when changing the face indices), contrary to a planar (or volumic) arrangement of monopole oscillators since in the latter case oscillators on the edges are not equivalent to the ones in the center. Another difference from separate wells in a planar arrangement [15] is that the resonance frequency decreases with the number of wells with a separate amount of gas since only the effective mass is increased by acoustic coupling. Here the frequency initially increases with $N$ because the effective stiffness also increases with the gas coupling, with the gas being shared by interfaces.

Monopole emission of a cubic bubble.-In order to specify the precise distribution of sound and verify the validity of our assumptions, we conducted 3D numerical simulations of sound propagation through the cubic bubbles. The simulations were implemented with a finite-difference time-domain (FDTD) resolution of the elastodynamic equation for both the fluid and the solid structure, based on a freely available software developed in our group [16]. We model the frame as a solid material (with properties close to that of Plexiglas), and water and air were modeled as perfect fluids. A simulation volume of $20 \times 20 \times 20 \mathrm{~mm}^{3}$ was meshed with a grid step of $100 \mu \mathrm{m}$, surrounded by perfectly matched layers to mimic propagation in an unbounded medium. Wideband pressure pulses in the kilohertz range ( $2 \mathrm{kHz}$ center frequency, 200\% bandwidth) were propagated with and without the presence of the cubic bubble, analogous to the experimental situation, to derive the resonant frequency of the bubble. The validity of our approach was first confirmed by simulating the response of spherical air bubbles of different sizes, which yielded values of the resonant frequency in excellent agreement with the value predicted by the Minnaert theory (accuracy better than $0.5 \%$ ). (See the Supplemental Material for further details [17].)

Our simulations confirmed the behavior observed experimentally for the effects of size [Fig. 2(b)] and the number of faces [Fig. 3(a)] while shedding light on the determinant parameters of the resonant frequency: it was observed by removing the solid frame (only possible with simulations) that the resonant frequency of a cubic air bubble is very close (typically less than $1 \%$ relative difference) to that of a spherical bubble of equivalent volume. It is therefore the presence of the solid frame which turns the cubic bubble into six coupled oscillators, each pinned to a rigid frame, as assumed by our analytical model. Other important information from the simulations is that cubic bubbles behave the same with monopole acoustic sources [Fig. 3(b)] as with spherical bubbles.

A side effect: Capillary surface modes.-At much larger amplitudes of acoustic pressure, we observed resonant surface modes with nodes and antinodes across the square interface (see Fig. 4, red arrow).

In order to reach such high acoustic pressures, we designed a specific setup: a steel tank with glass windows (see Fig. 1 in the Supplemental Material [17]), with a shaker pushing or pulling the water directly through a tight orifice. A precise map of the vibration amplitude of those surface modes was performed on a customized cube (see Fig. 2 in the Supplemental Material [17]). Such an elevation map is morphed on the top face of the image in Fig. 4. 


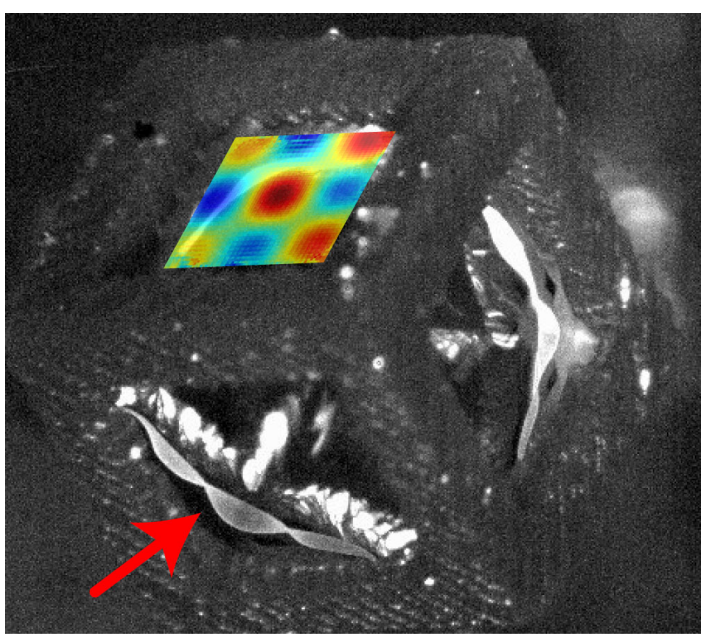

FIG. 4. Surface modes at $f=760 \mathrm{~Hz}$, for a cube of inner size $2 a=1.7 \mathrm{~mm}$. The red arrow shows the vibration of the reflection of the line of light. The image of a front measurement of the vibration amplitude performed on a single face is morphed and superimposed on the top face of the cube. It shows a standing wave with $3 \times 3$ half wavelengths. Here the interfaces were slightly pushed inside when closing the watertight tank. See also the movie in the Supplemental Material showing the vibration mode [17].

We interpret those modes as standing capillary waves. The modes are probably directly excited by the contact line on the edges, without any parametric instability. Note that these modes are not emissive: there is no signature for them in the frequency spectrum from hydrophone recordings.

Conclusion and perspectives.-We have shown that cubic bubbles are good candidates for the design of acoustic metamaterials: they are subwavelength resonators with a good quality factor and an isotropic emission field close to the one of spherical bubbles. Furthermore, they are easy to assemble in a large number using 3D stereolithography, allowing for stability in space and time. Because they have flat interfaces, there is no capillary overpressure that would tend to speed up dissolution. Moreover there is no rectified diffusion under oscillations that would inflate the gas volume, as is the case with spherical bubbles [18,19]. Future work will aim at understanding the interaction between a large number of cubic bubbles, paving the way to precisely designed acoustic metamaterials.

P.M. acknowledges financial support from the European Community's Seventh Framework Programme (FP7/2007-2013) ERC Grant Agreement Bubbleboost
No. 614655 and an anonymous referee for the suggestion to consider the liquid path to model the interaction between faces. M. H. acknowledges Léo Denat for support in the experiments, in addition to Benjamin Dollet, Thomas Combriat, Patrice Ballet, and Thibaut Metivet for the useful discussions.

*philippe.marmottant@univ-grenoble-alpes.fr

[1] M. Minnaert, Philos. Mag. 16, 235 (1933).

[2] T. G. Leighton, The Acoustic Bubble (Academic Press, London, 1994).

[3] V. Leroy, A. Strybulevych, M. G. Scanlon, and J. H. Page, Eur. Phys. J. E 29, 123 (2009).

[4] A. Bretagne, A. Tourin, and V. Leroy, Appl. Phys. Lett. 99, 221906 (2011).

[5] T. Brunet, A. Merlin, B. Mascaro, K. Zimny, J. Leng, O. Poncelet, C. Aristégui, and O. Mondain-Monval, Nat. Mater. 14, 384 (2015).

[6] S. Raffy, B. Mascaro, T. Brunet, O. Mondain-Monval, and J. Leng, Adv. Mater. 28, 1760 (2016).

[7] M. Lanoy, J. H. Page, G. Lerosey, F. Lemoult, A. Tourin, and V. Leroy, Phys. Rev. B 96, 220201(R) (2017).

[8] M. Lanoy, R. Pierrat, F. Lemoult, M. Fink, V. Leroy, and A. Tourin, Phys. Rev. B 91, 224202 (2015).

[9] T. L. Heath, The Works of Archimedes (Cambridge University Press, Cambridge, England, 1897).

[10] V. Leroy, M. Devaud, T. Hocquet, and J. C. Bacri, Eur. Phys. J. E 17, 189 (2005).

[11] P. S. Epstein and M. S. Plesset, J. Chem. Phys. 18, 1505 (1950).

[12] V. Leroy, Ph.D. thesis, Université Paris 7 - Denis Diderot, 2004.

[13] D. L. Miller and W. L. Nyborg, J. Acoust. Soc. Am. 73, 1537 (1983).

[14] H. Gelderblom, A. G. Zijlstra, L. van Wijngaarden, and A. Prosperetti, Phys. Fluids 24, 122101 (2012).

[15] H. Rathgen, K. Sugiyama, C.-D. Ohl, D. Lohse, and F. Mugele, Phys. Rev. Lett. 99, 214501 (2007).

[16] E. Bossy, simsonic, a FDTD simulation freeware, http:// www.simsonic.fr/.

[17] See Supplemental Material at http://link.aps.org/ supplemental/10.1103/PhysRevLett.123.254501 for a description of the high-pressure set-up and a movie of the interface oscillation, together with the methods used for 3D numerical simulations.

[18] M. M. Fyrillas and A. J. Szeri, J. Fluid Mech. 277, 381 (1994).

[19] W. Lauterborn and T. Kurz, Rep. Prog. Phys. 73, 106501 (2010). 